\title{
Treadmill exercise during pregnancy ameliorates post-traumatic stress disorder-induced anxiety-like responses in maternal rats
}

\author{
JIN-HEE SEO ${ }^{1}$, TAE-WOON KIM ${ }^{2}$, CHANG-JU KIM ${ }^{2}$, YUN-HEE SUNG ${ }^{3}$ and SAM-JUN LEE ${ }^{4}$ \\ ${ }^{1}$ Division of Sports Science, Baekseok University, Cheonan 330-704; ${ }^{2}$ Department of Physiology, College of Medicine, \\ Kyung Hee University, Seoul 130-701; ${ }^{3}$ Department of Physical Therapy, Kyungnam University, Changwon 631-701; \\ ${ }^{4}$ Department of Physical Education, College of Health, Welfare and Education, \\ Tong Myong University, Busan 608-711, Republic of Korea
}

Received August 9, 2012; Accepted November 20, 2012

DOI: $10.3892 / \mathrm{mmr} .2012 .1197$

\begin{abstract}
Post-traumatic stress disorder (PTSD) is an anxiety disorder triggered by life-threatening events that cause intense fear. Exercise is known to have protective effects on neuropsychiatric diseases. The present study investigated whether treadmill exercise during pregnancy reduced or alleviated symptoms of PTSD in maternal rats. To induce predator stress in pregnant rats, rats were exposed to a hunting dog in an enclosed room. Exposure time was three 10-min daily sessions separated by $1 \mathrm{~h}$, starting at week 1 of pregnancy until delivery. Pregnant rats in the exercise group were forced to run on a treadmill for 30 min once a day, starting one week following pregnancy until delivery. Rats receiving predator stress during pregnancy exhibited PTSD anxiety-like behaviors following delivery. Expression of 5-hydroxytryptamine (5-HT) and its synthesizing enzyme tryptophan hydroxylase (TPH) in the dorsal raphe was increased compared with unstressed rats. Expression of c-Fos and neuronal nitric oxide synthases (nNOS) in the hypothalamus and locus coeruleus were higher in the rats receiving stress during pregnancy compared with unstressed rats. By contrast, treadmill exercise during pregnancy ameliorated anxiety-like behaviors and reduced the expression of 5-HT, TPH, c-Fos and nNOS in the PTSD maternal rats. The results of the present study indicate that exercise during pregnancy is suitable for use as a therapeutic strategy to reduce anxiety-related disorders, including PTSD.
\end{abstract}

\section{Introduction}

Severe stress induces anxiety disorders which manifest as marked, continual, excessive and irrational reactions to fear (1).

Correspondence to: Professor Sam-Jun Lee, Department of Physical Education, College of Health, Welfare and Education, Tong Myong University, 428 Sinseonno, Nam-gu, Busan 608-711, Republic of Korea

E-mail: anada23@tu.ac.kr

Key words: post-traumatic stress disorder, pregnancy, treadmill exercise, anxiety, rats
Anxiety disorders are closely associated with an inability to distinguish between learned fear responses (2). Of the anxiety disorders, post-traumatic stress disorder (PTSD) exhibits a variety of symptoms that include exaggerated fear, helplessness and terror following extremely stressful events. The symptoms of the re-experience of an earlier traumatic event are panic attack, phobic avoidance of situations that resemble the traumatic event and psychic numbing (3). Pharmacological treatments of PTSD patients are mainly serotonergic agonists. Delayed onset, poor efficacy and relapse are the main complications of these drugs $(4,5)$.

Serotonin, also known as 5-hydroxytryptamine (5-HT), has been implicated in a wide variety of physiological, sensorimotor and behavioral functions. Malfunction of the serotonergic systems has been hypothesized to be the main etiological factor for numerous psychiatric illnesses, including anxiety, mood and eating disorders, as well as neurovascular disorders, i.e., migraine $(6,7)$. Serotonergic systems inhibit proactive coping responses, including aggression and escape behaviors, and facilitate passive-submissive behaviors including fear- and anxiety-like behaviors $(6,8,9)$. The biosynthesis of 5-HT from tryptophan is catalyzed by tryptophan hydroxylase (TPH), initially producing 5-hydroxytryptophan. The decarboxylation of 5-hydroxytryptophan into 5-HT is catalyzed by an aromatic amino acid decarboxylase. As $\mathrm{TPH}$ is the rate-limiting enzyme in serotonin production, its expression is used as the indicator of serotonin synthesis (10). Depressed suicide victims have higher TPH immunoreactivity in the dorsal raphe nucleus compared with nonpsychiatric control subjects (11). Chamas et al (12) proposed that repeated immobilization stress increases TPH mRNA and protein concentrations.

The immediate-early gene product, c-Fos, is expressed throughout the brain in response to a variety of stimuli. The detection of c-Fos is a powerful tool for the study of intracellular responses of neurons. As c-Fos expression in the brain represents neuronal activation, increased c-Fos expression indicates that neurons are activated by stimuli (13-15). Increased serotonergic neuronal activity induced by social defeat is followed by increased c-Fos expression, resulting in an increased extracellular serotonin concentration within the dorsal raphe nucleus $(8,16)$. 
The biological substrates involved in social interactions and anxiety behaviors remain unclear. Nitric oxide (NO) has been implicated in social interactions and anxiety-like behaviors $(17,18)$. NO is synthesized from L-arginine by a family of isoformic enzymes known as NO synthases (NOSs). Three isoforms of NOS have been identified: neuronal (nNOS), endothelial (eNOS) and inducible (iNOS). Of these, nNOS is rich throughout the limbic system of the brain area, which controls emotional behaviors $(19,20)$. Inhibition of nNOS alters social interactions and anxiety-like behaviors $(17,18)$ and inhibition of NOS causes antidepressive effects (21).

Exercise is known to enhance learning ability and memory function (22-24). An ameliorating effect of exercise on depression has also been reported (25-28). However, the effects of exercise on stress-induced anxiety symptoms remain unclear. In the present study, the effect of treadmill exercise on anxiety-like symptoms induced by stress during pregnancy was investigated in maternal rats. The anxiolytic effects of treadmill exercise were evaluated by an elevated plus maze (EPM) test and the expression of 5-HT, TPH, c-Fos and nNOS were determined by immunohistochemistry.

\section{Materials and methods}

Animals. Adult pregnant Sprague-Dawley rats were obtained from a commercial breeder (Orient Co., Seoul, Korea). The animals were housed under controlled temperature $\left(23 \pm 2^{\circ} \mathrm{C}\right)$ and lighting (08:00-20:00 h) conditions, with food and water available ad libitum. The pregnant rats were randomly divided into 4 groups ( $n=5$ per group): control, control exercise, PTSD-induced and PTSD-induced and exercise. All experimental procedures were performed in accordance with the animal care guidelines of the National Institutes of Health and the Korean Academy of Medical Sciences.

Induction of stress. Stress was induced by exposure of pregnant rats to a hunting dog in an enclosed room as previously described (29). Exposure time was $10 \mathrm{~min}$, repeated 3 times/day with a 1-h interval. Exposure of maternal rats to the hunting dog was performed from day 7 following pregnancy until delivery. The response of each pregnant rat during predator exposure involved observation from a distance, approaching and sniffing and chasing with occasional mild attack. The hunting dog was able to approach, but was not able to injure the pregnant rats. The same hunting dog was used throughout the experiment. Control rats were left undisturbed during the pregnancy.

Exercise protocol. Pregnant rats in the exercise groups were forced to run on a treadmill for $30 \mathrm{~min} /$ day starting at day 7 of pregnancy until delivery. The exercise load consisted of running at a speed of $2 \mathrm{~m} / \mathrm{min}$ for $5 \mathrm{~min}$, followed by $5 \mathrm{~m} / \mathrm{min}$ for $5 \mathrm{~min}$ and then $8 \mathrm{~m} / \mathrm{min}$ for the last $20 \mathrm{~min}$. The rats in the non-exercise groups were left on the treadmill without running for the same period as the exercise groups.

Elevated plus maze test. Following delivery, the maternal rats were left undisturbed together with their neonatal rats for 3 weeks. Anxiety-like behavior was evaluated using the EPM test as described previously $(30,31)$. The EPM consisted of two opposing open arms $(45 \times 10 \mathrm{~cm})$ and two closed arms $(45 \times 10 \times 50 \mathrm{~cm})$ that extended from a central platform $(10 \times 10 \mathrm{~cm})$ elevated $65 \mathrm{~cm}$ above the floor. Each pregnant rat was placed on the central platform facing a closed arm and was allowed to freely explore the maze for $5 \mathrm{~min}$. Entry into an arm was defined as entry of all four paws into the arm. Time spent in the open and closed arms were measured.

Brain preparation. Animals were sacrificed immediately following EPM testing. To prepare the brain slices, animals were fully anesthetized with Zoletil $50^{\circledR}(10 \mathrm{mg} / \mathrm{kg}$, i.p.; Vibac, Carros, France), transcardially perfused with $50 \mathrm{mM}$ phosphate-buffered saline (PBS) and then fixed with freshly prepared solution of $4 \%$ paraformaldehyde in $100 \mathrm{mM}$ phosphate buffer ( $\mathrm{pH}$ 7.4). Brains were then removed, post-fixed in the same fixative overnight and transferred into a $30 \%$ sucrose solution for cryoprotection. Coronal sections ( $40-\mu \mathrm{m}$ thick) were made using a freezing microtome (Leica Microsystems Ltd., Nussloch, Germany).

Immunohistochemistry for 5-HT and TPH.Immunohistochemistry for 5-HT- and TPH-positive cells in the dorsal raphe nucleus was conducted as previously described (28). The dorsal raphe nucleus spanning from Bregma -7.20 to $-8.00 \mathrm{~mm}$ were obtained from each brain. To begin the procedure, the sections were incubated in PBS for $10 \mathrm{~min}$ and then washed three times in the same buffer. Sections were then incubated in $1 \%$ hydrogen peroxide $\left(\mathrm{H}_{2} \mathrm{O}_{2}\right)$ for $30 \mathrm{~min}$. Next, the sections were incubated overnight with rabbit anti-5-HT (1:5,000; Immuno Star, Hudson, WI, USA) or mouse anti-TPH antibodies (1:1,000; Oncogene Research Products, Cambridge, UK). The sections were then incubated for $1 \mathrm{~h}$ with anti-rabbit secondary antibody (1:200; Vector Laboratories, Burlingame, CA, USA) for 5-HT immunohistochemistry or anti-mouse secondary antibody (1:200; Vector Laboratories) for TPH immunohistochemistry. Next, the sections were incubated with avidin-biotin-peroxidase complex $(1: 100$; Vector Laboratories) for $1 \mathrm{~h}$ at room temperature. For staining, the sections were incubated in a solution consisting of $0.02 \%$ 3,3'-diaminobenzidine tetrahydrochloride (DAB) and $0.03 \%$ $\mathrm{H}_{2} \mathrm{O}_{2}$ in $50 \mathrm{mM}$ Tris- $\mathrm{HCl}$ ( $\mathrm{pH} 7.6$ ) for $\sim 5 \mathrm{~min}$, following which they were washed with PBS and mounted onto gelatin-coated slides. The number of 5-HT- and TPH-positive cells in the dorsal raphe nucleus of the selected sections were counted using a light microscope (Olympus, Tokyo, Japan).

Immunohistochemistry for c-Fos and nNOS. Immunohistochemistry for c-Fos- and nNOS-positive cells in the hypothalamus (Bregma -1.80 to $-1.92 \mathrm{~mm}$ ) and locus coeruleus (Bregma -9.84 to $-9.96 \mathrm{~mm}$ ) was conducted as previously described $(13,32)$. To begin the procedure, sections were incubated in PBS for $10 \mathrm{~min}$ and then washed three times in the same buffer. The sections were then incubated in $1 \%$ $\mathrm{H}_{2} \mathrm{O}_{2}$ for $30 \mathrm{~min}$. Next, sections were incubated overnight with rabbit anti-c-Fos (1:500; Santa Cruz Biotechnology, Inc., Santa Cruz, CA, USA) or mouse anti-nNOS antibodies (1:500; BD Biosciences, San Jose, CA, USA). The sections were then incubated for $1 \mathrm{~h}$ with anti-rabbit secondary antibody (1:200; Vector Laboratories) for c-Fos immunohistochemistry and with anti-mouse secondary antibody (1:200; Vector Laboratories) 

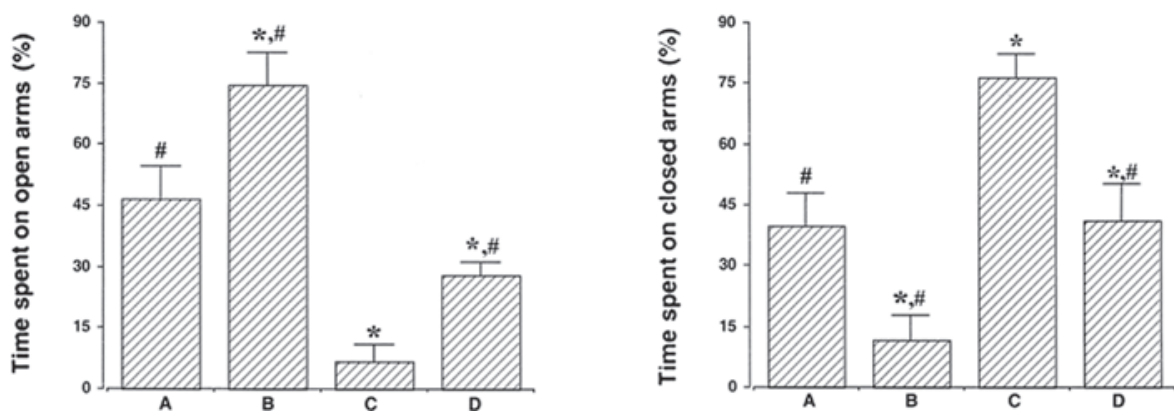

Figure 1. Effect of treadmill exercise on anxiety-like behavior in the elevated plus maze test. (A) Control, (B) control exercise, (C) PTSD-induced and (D) PTSD-induced and exercise groups. ${ }^{*} \mathrm{P}<0.05$, vs. control group. ${ }^{\#} \mathrm{P}<0.05$, vs. PTSD-induced group. Values are the mean $\pm \mathrm{SEM}$. PTSD, post-traumatic stress disorder.

for nNOS immunohistochemistry. Next, sections were incubated with avidin-biotin-peroxidase complex (1:100; Vector Laboratories) for $1 \mathrm{~h}$ at room temperature. For staining, the sections were incubated in a solution of $0.02 \% \mathrm{DAB}$ and $0.03 \%$ $\mathrm{H}_{2} \mathrm{O}_{2}$ in $50 \mathrm{mM}$ Tris- $\mathrm{HCl}(\mathrm{pH} 7.6)$ for $\sim 5 \mathrm{~min}$, washed with PBS and mounted onto gelatin-coated slides. The numbers of c-Fos and nNOS-positive cells in the hypothalamus and locus coeruleus of the selected sections were counted using a light microscope (Olympus).

Statistical analysis. Results are presented as the mean \pm SEM. Data were analyzed by one-way analysis of variance, followed by Duncan's post-hoc test using SPSS software (SPSS, Chicago, IL, USA). $\mathrm{P}<0.05$ was considered to indicate a statistically significant difference.

\section{Results}

Effect of treadmill exercise on anxiety-like behaviors. The effect of treadmill exercise on anxiety-like behaviors is presented in Fig. 1. The percentage of time spent on the open and closed arms during the total 5-min test were calculated. The open arm values were $46.47 \pm 8.20 \%$ in control, $74.72 \pm 7.93 \%$ in control exercise, $6.90 \pm 4.09 \%$ in PTSD-induced and $28.02 \pm 3.22 \%$ in the PTSD-induced and exercise group. The decreased value obtained for the PTSD-induced group compared with the control was found to be significant $(\mathrm{P}<0.05)$. By contrast, treadmill exercise increased the percentage of time spent in the open arms in the control and PTSD-induced groups $(\mathrm{P}<0.05)$.

The percentage of time spent in the closed arms was $39.97 \pm 8.04 \%$ in control, $11.57 \pm 6.27 \%$ in control exercise, $76.55 \pm 5.64 \%$ in PTSD-induced and $40.97 \pm 9.11 \%$ in the PTSD-induced and exercise group. The time spent in the closed arms was identified to be significantly increased in the PTSD-induced group compared with the control $(\mathrm{P}<0.05)$. By contrast, treadmill exercise was observed to significantly decrease the time spent in the closed arms in the control and PTSD-induced groups $(\mathrm{P}<0.05)$.

Effect of treadmill exercise on 5-HT and TPH expression in the dorsal raphe nucleus. Photomicrographs of 5-HT- and TPH-positive cells in the dorsal raphe nucleus are presented in Fig. 2. The number of 5-HT-positive cells was 180.50 \pm 6.93 in control, $208.00 \pm 9.56$ in control exercise, $320.00 \pm 16.26$ in
PTSD-induced and 247.60 \pm 6.66 in the PTSD-induced and exercise group. Expression of 5-HT in the dorsal raphe nucleus was found to be significantly increased in the PTSD-induced group compared with the control $(\mathrm{P}<0.05)$. Treadmill exercise suppressed 5-HT expression in the dorsal raphe nucleus of the PTSD-induced group $(\mathrm{P}<0.05)$. In normal rats, treadmill exercise slightly increased 5-HT expression in the dorsal raphe nucleus $(\mathrm{P}<0.05)$.

The number of TPH-positive cells was $262.00 \pm 15.46$ in control, $339.50 \pm 11.55$ in control exercise, 551.66 \pm 50.89 in PTSD-induced and 402.00 \pm 15.74 in the PTSD-induced and exercise group. TPH expression in the dorsal raphe nucleus was increased in the PTSD-induced group compared with control $(\mathrm{P}<0.05)$. Treadmill exercise suppressed TPH expression in the dorsal raphe nucleus of the PTSD-induced group $(\mathrm{P}<0.05)$. In normal rats, treadmill exercise slightly increased $\mathrm{TPH}$ expression in the dorsal raphe nucleus $(\mathrm{P}<0.05)$.

Effect of treadmill exercise on c-Fos expression in the hypothalamus and locus coeruleus. Photomicrographs of c-Fos-positive cells in the hypothalamus and locus coeruleus are presented in Fig. 3. The number of c-Fos-positive cells in the hypothalamus was $111.37 \pm 16.35$ in control, $113.50 \pm 8.15$ in control exercise, $170.00 \pm 8.47$ in PTSD-induced and $117.75 \pm 20.16$ in the PTSD-induced and exercise group. c-Fos expression in the hypothalamus was increased in the PTSD-induced group compared with control $(\mathrm{P}<0.05)$. Treadmill exercise suppressed c-Fos expression in the hypothalamus of the PTSD-induced group $(\mathrm{P}<0.05)$. In normal rats, treadmill exercise was identified to have no significant effect on c-Fos expression in the hypothalamus.

The number of c-Fos-positive cells in the locus coeruleus was $35.58 \pm 5.30$ in control, $56.40 \pm 6.33$ in control exercise, 71.53 \pm 7.34 in PTSD-induced and $37.78 \pm 5.96$ in the PTSD-induced and exercise group. c-Fos expression in the locus coeruleus was increased in the PTSD-induced group compared with the control $(\mathrm{P}<0.05)$. Treadmill exercise suppressed c-Fos expression in the locus coeruleus of the PTSD-induced group $(\mathrm{P}<0.05)$. In normal rats, treadmill exercise increased c-Fos expression in the locus coeruleus $(\mathrm{P}<0.05)$.

Effect of treadmill exercise on nNOS expression in the hypothalamus and locus coeruleus. Data concerning the nNOS-positive cells in the hypothalamus and locus coeruleus 
A

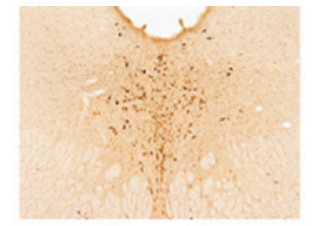

C

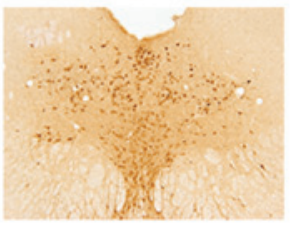

B

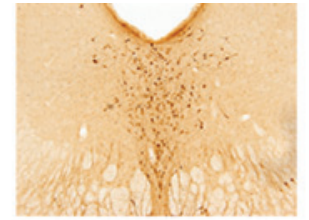

D

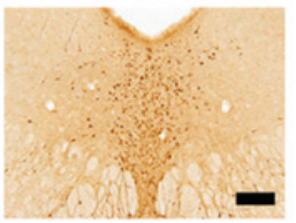

$\mathbf{F}$

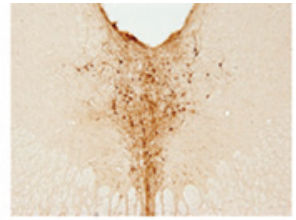

$\mathbf{H}$

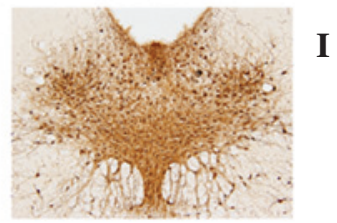

G

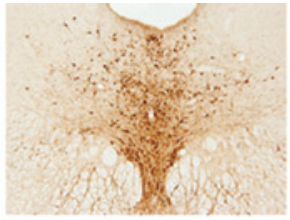

I

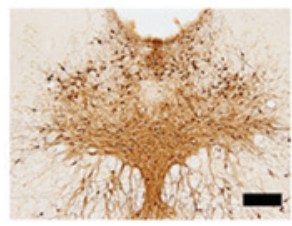

$\mathbf{E}$

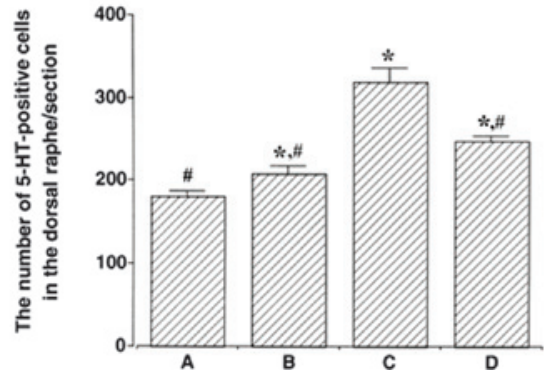

$\mathbf{J}$

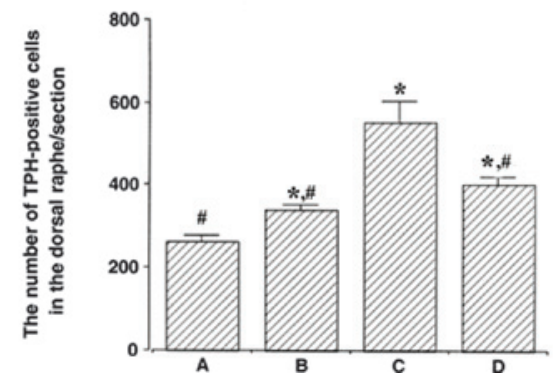

Figure 2. Effect of treadmill exercise on 5-HT and TPH expression in the dorsal raphe nucleus. Photomicrographs of (A-D) 5-HT- and (F-I) TPH-positive cells in the dorsal raphe nucleus. Scale bars represent $250 \mu \mathrm{m}$. Quantification of the number of (E) 5-HT- and (J) TPH-positive cells in the dorsal raphe nucleus in each group. (A and F) Control, (B and G) control exercise, (C and H) PTSD-induced and (D and I) PTSD-induced and exercise groups. "P<0.05, vs. control group. "P<0.05, vs. PTSD-induced group. Values are the mean \pm SEM. 5-HT, 5-hydroxytryptamine; TPH, tryptophan hydroxylase; PTSD, post-traumatic stress disorder.

A

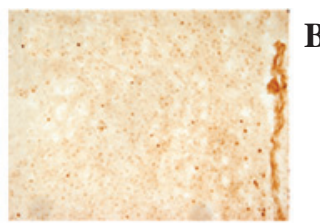

C

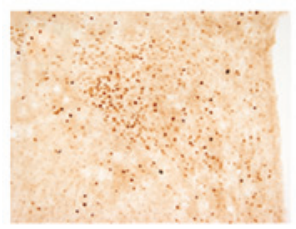

$\mathbf{E}$

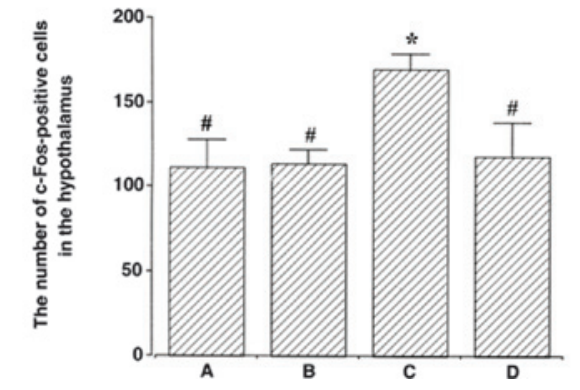

$\mathbf{F}$

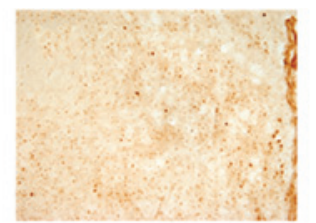

D

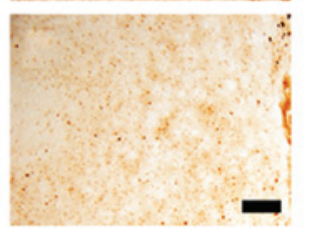

H

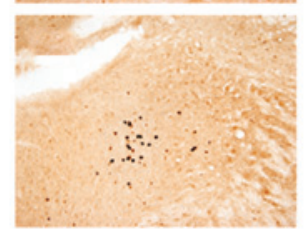

G

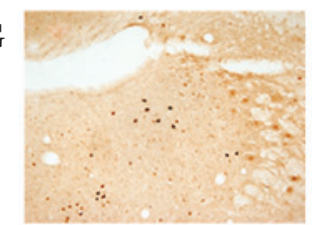

I

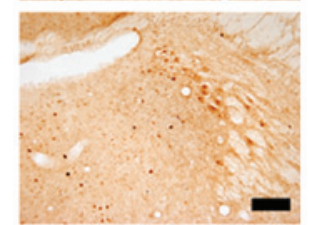

$\mathbf{J}$

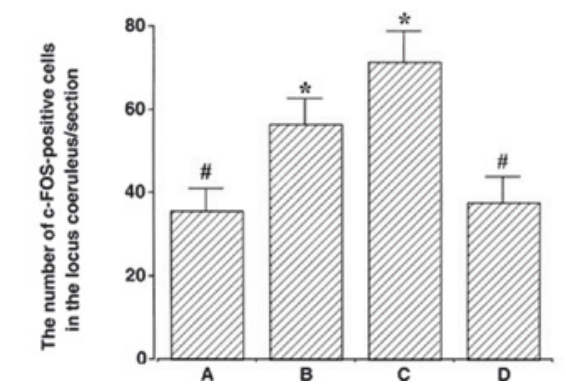

Figure 3. Effect of treadmill exercise on c-Fos-positive cells in the hypothalamus and locus coeruleus. Photomicrographs of c-Fos-positive cells in the (A-D) hypothalamus and (F-I) locus coeruleus. Scale bars represent $50 \mu \mathrm{m}$. (E and J) Quantification of the number of c-Fos-positive cells in each group. (A and F) Control, (B and G) control exercise, (C and H) PTSD-induced and (D and I) PTSD-induced and exercise groups. "P<0.05, vs. control group. "P $<0.05$, vs. PTSD-induced group. Values are the mean \pm SEM. PTSD, post-traumatic stress disorder.

are presented in Fig. 4. The number of nNOS-positive cells in the hypothalamus was $64.11 \pm 4.82$ in control, $63.66 \pm 4.13$ in control exercise, $103.64 \pm 4.17$ in PTSD-induced and 83.60 \pm 2.96 in the PTSD-induced and exercise group. nNOS expression in the hypothalamus was increased in the PTSD-induced group compared with the control $(\mathrm{P}<0.05)$. Treadmill exercise suppressed nNOS expression in the hypothalamus of the PTSD-induced group $(\mathrm{P}<0.05)$. In normal rats, treadmill exercise was found to have no significant affect on nNOS expression in the hypothalamus.

The number of nNOS-positive cells in the locus coeruleus was $64.11 \pm 4.82$ in control, $63.66 \pm 4.13$ in control exercise, $103.64 \pm 4.17$ in PTSD-induced and $83.60 \pm 2.96$ in the PTSD-induced and exercise group. nNOS expression 
A

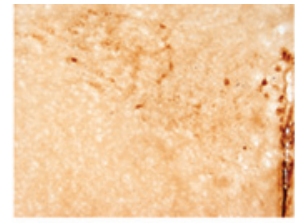

C

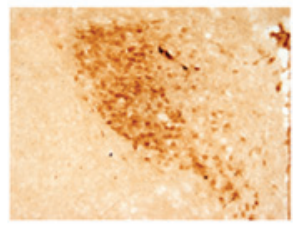

B

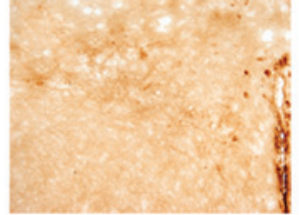

D

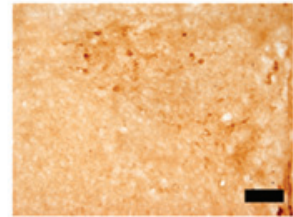

F

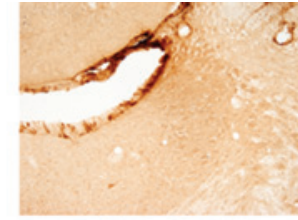

H

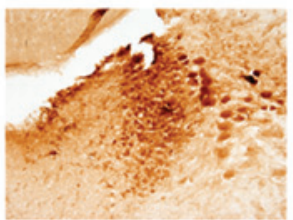

G
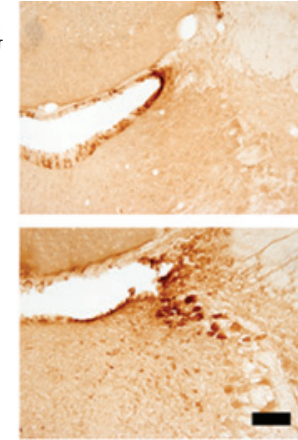
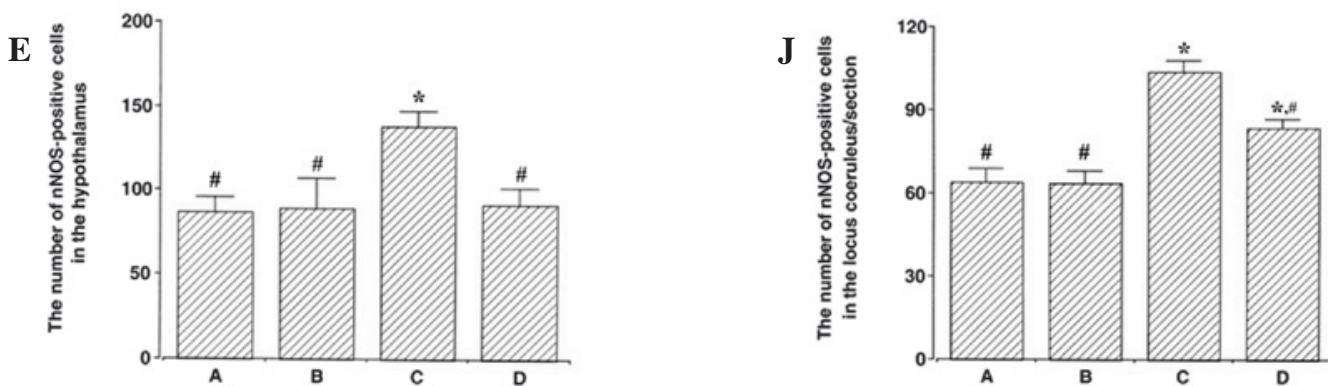

Figure 4. Effect of treadmill exercise on nNOS-positive cells in the hypothalamus and locus coeruleus. Photomicrographs of nNOS-positive cells in the (A-D) hypothalamus and (F-I) locus coeruleus. Scale bars represent $50 \mu \mathrm{m}$. (E and J) Quantification of the number of nNOS-positive cells in each group. (A and F) Control, (B and G) control exercise, (C and H) PTSD-induced and (D and I) PTSD-induced and exercise groups. ${ }^{*} \mathrm{P}<0.05$, vs. control group. ${ }^{*} \mathrm{P}<0.05$, vs. PTSD-induced group. Values are the mean \pm SEM. nNOS, neuronal nitric oxide synthase; PTSD, post-traumatic stress disorder.

in the locus coeruleus was increased in the PTSD-induced group compared with the control $(\mathrm{P}<0.05)$. Treadmill exercise suppressed nNOS expression in the locus coeruleus of the PTSD-induced group $(\mathrm{P}<0.05)$. In normal rats, treadmill exercise was identified to have no significant effect on nNOS expression in the locus coeruleus.

\section{Discussion}

Exercise is known to reduce symptoms of depression and anxiety (25-27). In a previous study, aerobic exercise was reported to reduce childhood PTSD, depression and anxiety (26). In addition, a study utilizing an animal model identified that wheel running reversed a long-lasting interference with shuttle box escape produced by uncontrollable stress (33). Other studies have documented the efficacy of aerobic exercise on depressive disorders to be comparable to antidepressant medication $(25,27)$. In the present study, the anxiolytic effect of treadmill exercise on PTSD was investigated through analysis of 5-HT, TPH, c-Fos and nNOS expression levels in the brain.

PTSD is characterized mainly by symptoms of re-experiencing, avoidance and hyperarousal as a consequence of catastrophic and traumatic events that are distinguished from ordinary stressful life events. The EPM test is an unconditioned test for anxiety in rodents in which animals exposed to stress spend a reduced duration of time in the open arms of the test and increased time in the closed arms $(30,31)$. In the present study, the rats in the PTSD-induced group were observed to spend less time in the open arms and more time in the closed arms compared with the rats in the control group. These results indicate that exposure to the hunting dog during pregnancy induced PTSD in the maternal rats following delivery. Treadmill exercise significantly increased the time spent in the open arms and decreased the time spent in the closed arms in the PTSD-induced group, demonstrating that treadmill exercise during pregnancy reduced the anxiety-like behaviors of the PTSD-induced maternal rats. Previously, treadmill running in rats was reported to reduce anxiety-like behavior in the EPM test without a significant change in total activity in the open field test (34).

5-HT neurons in the dorsal raphe nucleus have been implicated in the stress-induced changes in behavior. The dorsal raphe nuclei are the major source of 5-HT innervation of the forebrain and are critical for response to stress. 5-HT, as well as noradrenaline and $\gamma$-aminobutyric acid, are important neurotransmitters implicated in anxiety (35). In a competitive study, losing male Syrian hamsters exhibited increased c-Fos immunoreactivity in the dorsal raphe nucleus neurons compared with winners or controls, indicating that social stress activates 5-HT neurons in the dorsal raphe nucleus, reduces $5-\mathrm{HT}_{1 \mathrm{~A}}$ autoreceptor-mediated inhibition and induces hyperactivity of 5-HT neurons (36). Inescapable shock and social defeat increased extracellular levels of 5-HT within the dorsal raphe nucleus in rats (16). The number and density of serotonergic neurons was also found to be higher in suicide victims than controls (11). TPH is the rate-limiting enzyme in serotonin production and its expression is used as an indicator of 5-HT synthesis (10). A previous study found that levels of TPH immunoreactivity were higher in suicides than controls in the dorsal raphe nucleus but were not identified to be different in the median raphe nucleus (10). The expression of the 5- $\mathrm{HT}_{1 \mathrm{~A}}$ receptor in the dorsal raphe nucleus was increased following prolonged stress exposure, indicating that an increase in $5-\mathrm{HT}_{1 \mathrm{~A}}$ receptor levels in the dorsal raphe nucleus may be important in the pathogenesis 
of PTSD rats (37). Liu et al (38) reported that neuronal apoptosis by chronic stress induced PTSD, hypothesizing that the disease is associated with $5-\mathrm{HT}_{1 \mathrm{~A}}$ receptor activity. In the current study, the expression levels of 5-HT and TPH were increased in the dorsal raphe nucleus of the PTSD-induced rats, indicating that exposure to the predator during pregnancy caused stress, which led to PTSD in maternal rats following delivery. By contrast, treadmill exercise decreased the expression of 5-HT and TPH in the dorsal raphe nuclei of the PTSD-induced rats, indicating that treadmill exercise during pregnancy may alleviate the aforementioned stress. These observations are consistent with additional studies demonstrating that voluntary exercise reduces the incidence of stress-related psychiatric disorders in humans and prevents serotonin-dependent behavioral consequences of stress in rodents (39).

The primary component of the stress response is the activation of the hypothalamic-pituitary-adrenal axis. This axis is a feedback loop containing a complex set of direct effects and feedback interactions between the hypothalamus, pituitary and adrenal glands. The hypothalamic paraventricular nucleus receives direct and indirect inputs from the hippocampus and amygdala and is central to orchestration of the physiological response to stress $(40,41)$.

c-Fos expression has been used to map the pattern of neural activation following either a single or repeated exposure of aggressive interaction $(14,15,42)$. Expression of c-Fos following stress is highly region-specific, including the paraventricular nucleus and locus coeruleus, and is involved in adaptation to social stress (14). Noise-induced c-Fos mRNA expression in the paraventricular nucleus has been markedly correlated with levels of plasma adrenocorticotropin hormone (43). c-Fos expression in the paraventricular nucleus and locus coeruleus is increased by single restraint and these increments are attenuated by repeated restraint stress (44). Restraint stress enhances c-Fos expression in neurons of the paraventricular nucleus, locus coeruleus, supraoptic nucleus, rostral raphe pallidus, nucleus of the solitary tract and ventrolateral medulla (45). In the current study, expression of c-Fos in the hypothalamic paraventricular nucleus and locus-related stress during pregnancy led to neuronal activation in stress-related areas of the brain. By contrast, treadmill exercise decreased the expression of c-Fos in the PTSD-induced rats, indicating that treadmill exercise during pregnancy suppressed neuronal activation induced by the stress exposure. These results are consistent with previous observations that uncontrollable stress increased c-Fos expression in the dorsal raphe nucleus and wheel running attenuated uncontrollable stress-induced c-Fos expression in the dorsal raphe nucleus (39).

A number of studies have indicated that NOS is involved in anxiety-related behaviors $(46,47)$. nNOS overexpression in the hippocampus is essential for chronic stress-induced depression and inhibition of nNOS signaling in the brain may represent a novel approach for the treatment of depressive disorders (48). Inhibition of nNOS was previously reported to prevent $N$-methyl-D-aspartate receptor activation-induced anxiogenic-like effect in mice (46). In the present study, expression of NOS in the hypothalamic paraventricular nucleus and locus coeruleus were increased in PTSD-induced rats, demonstrating that exposure to the hunting dog during pregnancy induced anxiety in the maternal rats following delivery. By contrast, treadmill exercise decreased the expression of NOS in PTSD-induced rats, indicating that treadmill exercise during pregnancy evoked an anxiolytic effect against stress exposure. Workman et al (47) demonstrated that nNOS inhibition reduced anxiety-like responses, concluding that NO may be important for mediating the effects of social interactions on anxiety.

In conclusion, exposure to a predator during pregnancy induced anxiety-like behaviors with enhancement of anxiety-related neurochemical parameters, including 5-HT, TPH, c-Fos and nNOS, in the brains of maternal rats following delivery. Treadmill exercise during pregnancy had an anxiolytic effect that alleviated the anxiety-induced increase of 5-HT, TPH, c-Fos and nNOS expression in the brain of the maternal rats. The present results are consistent with the hypothesis that exercise during pregnancy may be suitable as a therapeutic strategy to reduce anxiety-related disorders, including PTSD.

\section{Acknowledgements}

The present study was supported by the National Research Foundation of Korea funded by the Korean Government (NRF-2010-327-G00123).

\section{References}

1. Cryan JF and Holmes A: The ascent of mouse: advances in modelling human depression and anxiety. Nat Rev Drug Discov 4: 775-790, 2005.

2. Myers KM and Davis M: Mechanisms of fear extinction. Mol Psychiatry 12: 120-150, 2007.

3. Yehuda R: Post-traumatic stress disorder. N Engl J Med 346: 108-114, 2002.

4. Baker DG, Nievergelt CM and Risbrough VB: Post-traumatic stress disorder: emerging concepts of pharmacotherapy. Expert Opin Emerg Drugs 14: 251-272, 2009.

5. Davis LL, Frazier EC, Williford RB and Newell JM: Long-term pharmacotherapy for post-traumatic stress disorder. CNS Drugs 20: 465-476, 2006.

6. Bannai M,Fish EW,Faccidomo S and Miczek KA: Anti-aggressive effects of agonists at $5-\mathrm{HT}_{\mathrm{IB}}$ receptors in the dorsal raphe nucleus of mice. Psychopharmacology (Berl) 193: 295-304, 2007.

7. Barnes NM and Sharp T: A review of central 5-HT receptors and their function. Neuropharmacology 38: 1083-1152, 1999.

8. Cooper MA, McIntyre KE and Huhman KL: Activation of 5-HT autoreceptors in the dorsal raphe nucleus reduces the behavioral consequences of social defeat. Psychoneuroendocrinology 33: 1236-1247, 2008.

9. Graeff FG and Zangrossi H Jr: The dual role of serotonin in defense and the mode of action of antidepressants on generalized anxiety and panic disorders. Cent Nerv Syst Agents Med Chem 10: 207-217, 2010.

10. Boldrini M, Underwood MD, Mann JJ and Arango V: More tryptophan hydroxylase in the brainstem dorsal raphe nucleus in depressed suicides. Brain Res 1041: 19-28, 2005.

11. Underwood MD, Khaibulina AA, Ellis SP, Moran A, Rice PM, Mann JJ and Arango V: Morphometry of the dorsal raphe nucleus serotonergic neurons in suicide victims. Biol Psychiatry 46: 473-483, 1999 .

12. Chamas FM, Underwood MD, Arango V, Serova L, Kassir SA, Mann JJ and Sabban EL: Immobilization stress elevates tryptophan hydroxylase mRNA and protein in the rat raphe nuclei. Biol Psychiatry 55: 278-283, 2004.

13. Kim SU, Ko IG, Kim BK, et al: Transplantation of human adipose-derived stem cells into the urethra ameliorates stress urinary incontinence and blunts the induction of c-Fos immunoreactivities in brain areas related to micturition in female rats. Anim Cells Syst 14: 237-244, 2010. 
14. Martinez M, Phillips PJ and Herbert J: Adaptation in patterns of c-fos expression in the brain associated with exposure to either single or repeated social stress in male rats. Eur J Neurosci 10: 20-33, 1998

15. Weinberg MS, Girotti M and Spencer RL: Restraint-induced fra-2 and c-fos expression in the rat forebrain: relationship to stress duration. Neuroscience 150: 478-486, 2007.

16. Amat J, Aleksejev RM, Paul E, Watkins LR and Maier SF: Behavioral control over shock blocks behavioral and neurochemical effects of later social defeat. Neuroscience 165 : 1031-1038, 2010

17. Forestiero D, Manfrim CM, Guimarães FS and de Oliveira RM: Anxiolytic-like effects induced by nitric oxide synthase inhibitors microinjected into the medial amygdala of rats Psychopharmacology (Berl) 184: 166-172, 2006.

18. Pokk P and Väli M: The effects of the nitric oxide synthase inhibitors on the behaviour of small-platform-stressed mice in the plus-maze test. Prog Neuropsychopharmacol Biol Psychiatry 26 : 241-247, 2002

19. Bredt DS, Glatt CE, Hwang PM, Fotuhi M, Dawson TM and Snyder SH: Nitric oxide synthase protein and mRNA are discretely localized in neuronal populations of the mammalian CNS together with NADPH diaphorase. Neuron 7: 615-624, 1991

20. Dawson TM, Bredt DS, Fotuhi M, Hwang PM and Snyder SH Nitric oxide synthase and neuronal NADPH diaphorase are identical in brain and peripheral tissues. Proc Natl Acad Sci USA 88: 7797-7801, 1991

21. Jesse CR, Bortolatto CF, Savegnago L, Rocha JB and Nogueira CW: Involvement of L-arginine- nitric oxide-cyclic guanosine monophosphate pathway in the antidepressant-like effect of tramadol in the rat forced swimming test. Prog Neuropsychopharmacol Biol Psychiatry 32: 1838-1843, 2008.

22. Colcombe $\mathrm{S}$ and Kramer AF: Fitness effects on the cognitive function of older adults: a meta-analytic study. Psychol Sci 14: 125-130, 2003

23. Galper DI, Trivedi MH, Barlow CE, Dunn AL and Kampert JB Inverse association between physical inactivity and mental health in men and women. Med Sci Sports Exerc 38: 173-178, 2006.

24. Rovio S, Kåreholt I, Helkala EL, Viitanen M, Winblad B, Tuomilehto J, Soininen H, Nissinen A and Kivipelto M Leisure-time physical activity at midlife and the risk of dementia and Alzheimer's disease. Lancet Neurol 4: 705-711, 2005.

25. Blumenthal JA, Babyak MA, Doraiswamy PM, et al: Exercise and pharmacotherapy in the treatment of major depressive disorder. Psychosom Med 69: 587-596, 2007.

26. Newman CL and Motta RW: The effects of aerobic exercise on childhood PTSD, anxiety and depression. Int J Emerg Ment Health 9: 133-158, 2007.

27. Perraton LG, Kumar S and Machotka Z: Exercise parameters in the treatment of clinical depression: a systematic review of randomized controlled trials. J Eval Clin Pract 16: 597-604, 2010

28. Sung YH, Shin MS, Cho S, Baik HH, Jin BK, Chang HK, Lee EK and Kim CJ: Depression-like state in maternal rats induced by repeated separation of pups is accompanied by a decrease of cell proliferation and an increase of apoptosis in the hippocampus. Neurosci Lett 470: 86-90, 2010.

29. Adamec R, Walling S and Burton P: Long-lasting, selective, anxiogenic effects of feline predator stress in mice. Physiol Behav 83: 401-410, 2004.

30. Kung JC, Chen TC, Shyu BC, Hsiao S and Huang AC: Anxiety- and depressive-like responses and c-fos activity in preproenkephalin knockout mice: oversensitivity hypothesis of enkephalin deficit-induced posttraumatic stress disorder. J Biomed Sci 17: 29-43, 2010.
31. Sterley TL, Howells FM and Russell VA: Effects of early life trauma are dependent on genetic predisposition: a rat study. Behav Brain Funct 7: 11-25, 2011.

32. Kim EK, Lee MH, Kim H, et al: Maternal ethanol administration inhibits 5-hydroxytryptamine synthesis and tryptophan hydroxylase expression in the dorsal raphe of rat offspring. Brain Dev 27: 472-476, 2005.

33. Greenwood BN, Strong PV, Dorey AA and Fleshner M: Therapeutic effects of exercise: wheel running reverses stress-induced interference with shuttle box escape. Behav Neurosci 121: 992-1000, 2007.

34. Fulk LJ, Stock HS, Lynn A, Marshall J, Wilson MA and Hand GA: Chronic physical exercise reduces anxiety-like behavior in rats. Int J Sports Med 25: 78-82, 2004.

35. Sandford JJ, Argyropoulos SV and Nutt DJ: The psychobiology of anxiolytic drugs. Part 1: Basic neurobiology. Pharmacol Ther 88: 197-212, 2000.

36. Cooper MA, Grober MS, Nicholas CR and Huhman KL: Aggressive encounters alter the activation of serotonergic neurons and the expression of 5-HT $\mathrm{HRNA}_{1 \mathrm{~A}}$ in the hamster dorsal raphe nucleus. Neuroscience 161: 680-690, 2009.

37. Luo FF, Han F and Shi YX: Changes in $5-\mathrm{HT}_{1 \mathrm{~A}}$ receptor in the dorsal raphe nucleus in a rat model of post-traumatic stress disorder. Mol Med Rep 4: 843-847, 2011.

38. Liu H, Wang HT, Han F and Shi YX: Activity of 5-HT receptor is involved in neuronal apoptosis of the amygdala in a rat model of post-traumatic stress disorder. Mol Med Rep 4: 291-295, 2011

39. Greenwood BN, Foley TE, Burhans D, Maier SF and Fleshner M: The consequences of uncontrollable stress are sensitive to duration of prior wheel running. Brain Res 1033: 164-178, 2005.

40. Herman JP, Ostrander MM, Mueller NK and Figueiredo H: Limbic system mechanisms of stress regulation: hypothalamopituitary-adrenocortical axis. Prog Neuropsychopharmacol Biol Psychiatry 29: 1201-1213, 2005.

41. Jankord R and Herman JP: Limbic regulation of hypothalamo-pituitary-adrenocortical function during acute and chronic stress. Ann NY Acad Sci 1148: 64-73, 2008

42. O'Mahony CM, Sweeney FF, Daly E, Dinan TG and Cryan JF: Restraint stress-induced brain activation patterns in two strains of mice differing in their anxiety behaviour. Behav Brain Res 213: $148-154,2010$.

43. Burow A, Day HE and Campeau S: A detailed characterization of loud noise stress: intensity analysis of hypothalamo-pituitary-adrenocortical axis and brain activation. Brain Res 1062: 63-73, 2005.

44. Kwon MS, Seo YJ, Shim EJ, Choi SS, Lee JY and Suh HW: The effect of single or repeated restraint stress on several signal molecules in paraventricular nucleus, arcuate nucleus and locus coeruleus. Neuroscience 142: 1281-1292, 2006.

45. Goebel M, Stengel A, Wang L and Taché Y: Restraint stress activates nesfatin-1-immunoreactive brain nuclei in rats. Brain Res 1300: 114-124, 2009.

46. Miguel TT and Nunes-de-Souza RL: Anxiogenic-like effects induced by NMDA receptor activation are prevented by inhibition of neuronal nitric oxide synthase in the periaqueductal gray in mice. Brain Res 1240: 39-46, 2008.

47. Workman JL, Trainor BC, Finy MS and Nelson RJ: Inhibition of neuronal nitric oxide reduces anxiety-like responses to pair housing. Behav Brain Res 187: 109-115, 2008.

48. Zhou QG, Hu Y, Hua Y, Hu M, Luo CX, Han X, Zhu XJ, Wang B, Xu JS and Zhu DY: Neuronal nitric oxide synthase contributes to chronic stress induced depression by suppressing hippocampal neurogenesis. J Neurochem 103: 1843-1854, 2007. 